\title{
FIABILIDAD Y VALIDEZ DE UN INSTRUMENTO QUE UTILIZA FOTOGRAFÍAS PARA MEDIR LA HABILIDAD DE DIAGNÓSTICO DIFERENCIAL DE LESIONES CAUSADAS POR PRESIÓN Y/O POR INCONTINENCIA
}

\author{
RELIABILITY AND VALIDITY OF AN INSTRUMENT \\ THAT USES PHOTOGRAPHS TO MEASURE THE ABILITY \\ FOR DIFFERENTIAL DIAGNOSIS OF INJURIES CAUSED BY \\ PRESSURE AND/OR INCONTINENCE
}

\author{
HeIdi Hevia * \\ José GONZÁlez ${ }^{* *}$ \\ FranCISCA VIVEROS ${ }^{* * *}$ \\ CARIMe COFrÉ ${ }^{* * * *}$ \\ Tiare Donoso ${ }^{* * * *}$ \\ IgNaCIA OLIVARes ${ }^{* * * * *}$ \\ MELISA SHIELDS ${ }^{* * * * * *}$
}

\section{RESUMEN}

Objetivo: Evaluar la fiabilidad y validez de un instrumento que permite medir la habilidad visual para identificar y clasificar Dermatitis Asociada a Incontinencia (DAI), Ulceras por Presión (UPP) y lesiones mixtas, por parte de profesionales de enfermería. Material y método: Se aplicó un instrumento autoadministrado conformado por 14 fotografías a una muestra por conveniencia de 28 profesionales de enfermería, donde se debía identificar la lesión y luego clasificar su estado de avance. A modo exploratorio se calculó la fiabilidad anidada con Alpha de Cronbach y la validez con Análisis de Componentes Principales (ACP). Resultados: La fiabilidad inicial de las 14 fotografías (Alpha de Cronbach= 0,174) fue baja y tras eliminar 7 la fiabilidad fue aceptable (Alpha de Cronbach $=0,709$ ). Se estableció la presencia de dos dimensiones: por un lado, la habilidad de diagnosticar DAI que explica un 35\% de la varianza y, por otro, la habilidad de diagnosticar UPP, que explica el 22\% de la varianza, ya sea que se encuentren separadas o juntas en un mismo paciente. Las lesiones DAI son diagnosticadas en mayor proporción que las lesiones mixtas. Conclusión: El estudio aportó 6 fotografías fiables y válidas que pueden ser utilizadas en futuros trabajos para construir un instrumento más robusto que pueda ser utilizado en Chile para la capacitación en el área. La muestra estudiada tiene fortalezas en el diagnóstico de DAI y dificultades en el diagnóstico de lesiones mixtas.

Palabras clave: Confiabilidad y validez, fotografías, úlcera por presión, diagnóstico diferencial, incontinencia fecal.

\footnotetext{
*Enfermera. MSc. Enfermería. Facultad de Enfermería. Universidad Andrés Bello Campus Viña del Mar. Chile. Email: hmheviac@vtr.net.

${ }^{* *}$ Profesor de matemática y computación. PhD. Estadística. Facultad de Ciencias Naturales y Exactas. Universidad de Playa Ancha. Valparaíso. Email: jgonzalez@upla.cl

${ }^{* * *}$ Socióloga. Universidad de Los Lagos. Convenio Marco Formación Inicial Docente ULA 1758. Osorno. Chile. Email: f.viverosr@gmail.com

${ }^{* * * *}$ Enfermera. Enfermera suplente Unidad de Mediana Complejidad Médica. Hospital Carlos Van Buren. Valparaíso. Chile. Email: c.cofreganahin@gmail.com

${ }^{* * * * *}$ Enfermera. Clínica de Medicina Hospital Naval Almirante Neff. Viña del Mar. Chile. Email: tiara.donoso93@gmail.com ${ }^{* * * * * *}$ Enfermera. Unidad Médica Agudos (UMA). Hospital Carlos Van Buren. Valparaíso. Chile. Email: igna.olivares@hotmail.com

${ }_{* * * * * * *}^{*}$ Enfermera. Unidad de Paciente Crítico. Clínica Los Carrera. Quilpué. Chile. Email: meli_asm@hotmail.com
} 


\section{ABSTRACT}

Aim: To evaluate the reliability and validity of an instrument that allows to measure the visual ability to identify and classify Incontinence Associated Dermatitis (IAD), Pressure Ulcers (PU) and mixed lesions, by nursing professionals. Material and method: A self-administered instrument consisting of 14 photographs was applied to a convenience sample of 28 nurses. A set of 14 photographs were shown in which they were asked to identify and then classify the state of the lesion. In an exploratory manner, the nested reliability was calculated with Cronbach's Alpha and the validity with Principal Component Analysis (PCA). Results: The initial reliability of the 14 photographs was low (Cronbach Alpha $=0.174$ ), after removing 7 photographs the reliability became acceptable (Cronbach Alpha $=0.709$ ). The presence of two dimensions was established: on the one hand the ability to diagnose IAD, which explains $35 \%$ of the variance, and secondly, the ability to diagnose PU, which explains $22 \%$ of the variance, whether they are separated or together in the same patient. IAD is better diagnosed than mixed lesions. Conclusion: The study provided 6 reliable and valid photos that can be used in future work to build a more robust instrument that can be used in Chile for training in the area. The studied sample shows strengths when diagnosing IAD and difficulties when diagnosing mixed lesions.

Key words: Validity and reliability, photographs, pressure ulcer, diagnosis, differential, fecal incontinence.

Fecha recepción: 27/10/16 Fecha aceptación: 14/12/17

\section{INTRODUCCIÓN}

La National Pressure Ulcer Advisory Panel (NPUAP) y la European Pressure Ulcer Advisory Panel (EPUAP) expresan que la lesión Dermatitis Asociada a Incontinencia (DAI) es una condición que debe ser diferenciada de la Úlcera por Presión (UPP) o de una laceración de la piel, ya que comúnmente se confunde su diagnóstico (1-4).

Para facilitar la diferenciación entre las lesiones DAI y las UPP, la EPUAP establece criterios para su evaluación visual y las características a considerar son: causa que origina el daño, color, localización, profundidad, bordes, forma y distribución de las lesiones, presencia de tejido necrótico, exudados, factores asociados y síntomas como dolor, ardor y prurito $(1,3,5,6)$. En la práctica, el diagnóstico de estas lesiones es efectuado por enfermeras clínicas, para lo cual se requiere tener conocimientos relevantes que permitan distinguir las lesiones (7-10).

En general, la investigación respecto a la capacidad de diferenciación, por parte de profesionales de enfermería, se ha realizado utilizando fotografías (11-14). Baumgarten et al. (14) recomiendan que éstas sean tomadas de manera similar en términos de calidad y condiciones ambientales. Esto quiere decir que deben exponer claramente la zona comprometida sin intervenciones de artículos de curaciones, sábanas o pañales, con el fin de procurar de que quien observe, fije la atención en la lesión y no se distraiga en observar otros elementos o artefactos.

En Chile existen pocos estudios respecto a las habilidades para diferenciar las lesiones. Uno de ellos corresponde al estudio realizado por Hevia et al. (15) que evaluaron las competencias visuales para identificar y clasificar 14 lesiones en la zona sacra (DAI, UPP y lesiones mixtas) en un grupo de 46 enfermeras y estudiantes de enfermería. El grupo alcanzó una media de 8 puntos correctos (2 DE), por lo que fue evidente la dificultad que tuvieron para diferenciar las lesiones. Particularmente, en el caso de la clasificación correcta de las lesiones en diferentes estadios, se registraron muy bajos niveles, lo cual da cuenta de que la clasificación es una habilidad compleja que requiere entrenamiento y tiempo para ser desarrollada y los expertos recomiendan 
que estos tópicos deben ser incorporados en la formación de base del pregrado de enfermería (11).

Está claro, entonces, que en la práctica clínica de profesionales de enfermería, hay confusión en el diagnóstico de la DAI y la UPP $(1-4,16)$, razón que amerita la utilización de instrumentos que permitan desarrollar la capacidad de diagnosticar estas lesiones.

Según lo anterior, el objetivo de este estudio fue: evaluar la fiabilidad y validez de un instrumento que permite medir la habilidad visual para diferenciar lesiones DAI, UPP y mixtas.

\section{MATERIAL Y MÉTODO}

Tipo de estudio: Se realizó un estudio exploratorio implementado en un diseño no experimental.

Unidad de análisis y muestra: Ante la dificultad para acceder a una mayor representatividad, se consideró una muestra no probabilística por conveniencia, conformada por 28 inscritos en un programa de especialización en heridas, todos ellos profesionales de enfermería de la comuna de Viña del Mar, Chile.

Instrumento recolector de datos: Para medir las competencias visuales en la diferenciación de lesiones se aplicó un cuestionario autoadministrado que contenía las 14 imágenes seleccionadas que muestran 5 lesiones DAI, 4 lesiones UPP y 5 lesiones por causa mixta (UPP y DAI), cada una impresa a color, en alta resolución, sin edición y en tamaño carta. Se utilizó como referencia lo aplicado anteriormente por Hevia et al. (15), que está basado en la técnica de valoración visual utilizada por Grey et al. $(7,8)$ y la EPUAP (12). Dos enfermeras especialistas en heridas confirmaron que cada una de las fotografías correspondía efectivamente a los tipos de lesiones escogidas con sus respectivos esta- dios de avance. La habilidad de diagnóstico diferencial se midió en dos etapas: primero, se debía identificar el tipo de lesión (DAI, UPP o mixta) que mostraba la fotografía y después se debía clasificar el estadio de la lesión. Las respuestas se codificaron en correctas (valor 1) e incorrectas (valor 0). En caso de contestar correctamente la clasificación y no así la identificación, se consideró errónea la respuesta ya que indica escasa habilidad visual para diagnosticar diferenciadamente los tipos de lesiones.

Procesamiento y análisis de datos: Los datos se procesaron en el programa estadístico SPSS 22. Dado que el diagnóstico diferencial comprende la identificación y luego la clasificación correcta, se aplicó la fiabilidad anidada $(17,18)$, es decir, se realizó el cálculo del estadístico Alpha de Cronbach en dos momentos, a saber, para la identificación correcta y la clasificación correcta. Luego, para establecer la validez de las fotografías se trabajó con el Análisis de Componentes Principales (ACP) para variables categóricas, tipo de análisis factorial que cuantifica la agrupación de elementos en torno a una dimensión (19, 20). Finalmente, para evaluar la dificultad de las fotografías se utilizó el coeficiente de dificultad de González et al. (21), que unifica la cantidad de información y la consistencia del ítem (en este caso fotografías), en relación al test, expresando la dificultad en un valor que oscila entre 0 y 1 , mientras más cercano a 1 , mayor dificultad.

Aspectos éticos: Se aseguró el respeto de los derechos de las personas que participaron en el estudio. En el caso de los pacientes fotografiados, se les solicitó directamente y también a sus familias la autorización para tomar la fotografía, la cual sólo muestra la zona afectada. En el caso de los profesionales de enfermería, se explicó las implicancias del estudio a través de una carta de consentimiento informado, la cual fue firmada por todos los profesionales evaluados. Adicionalmente el 
estudio contó con la aprobación del Comité de Ética Científico de la Facultad de Enfermería de la Universidad Andrés Bello.

\section{RESULTADOS}

Características de la muestra: Los profesionales participantes del estudio fueron principalmente mujeres $(96,4 \%)$ con una edad promedio de 34,3 años (DE 9,1) y un rango entre 24 y 58. Los años de experiencia laboral fueron muy disímiles, con un promedio de 8,5 años (DE 8,7), con profesionales de hasta 35 años de experiencia laboral. De las 28 unidades muestrales, 22 profesionales $(78,6 \%)$ habían recibido por lo menos 1 capacitación previa en heridas entre el año 2007 y 2015.

Fiabilidad: En relación a la identificación correcta de las lesiones, se observó que la fiabilidad inicial con 14 imágenes fue de 0,174 (Tabla 1). De las 14 fotografías iniciales se eliminaron 7 (fotografías 2, 3, 4, 6, 9, 11 y 12), debido a su escaso poder discriminante. Con las 7 fotografías elegidas el estadístico Alpha de Cronbach aumentó a 0,677. Dado que el diagnóstico comprende la habilidad de identificar y clasificar correctamente, se trabajó con respuestas anidadas, lo cual requirió un nuevo cálculo de la fiabilidad (16, 17). Se recalculó el coeficiente Alpha para las respuestas de clasificación correctas, observándose que este Alpha aumentó al eliminar dos elementos: Fotografías 2 y 8 . Para evitar la excesiva reducción de fotografías, se decidió mantener la foto 8 y sólo eliminar la foto 2 ya que la foto 8 aumentaba escasamente la fiabilidad $(0,016)$, mientras que la eliminación de la foto 2 aumentó la fiabilidad en 0,043 puntos. La foto 8 es la única que muestra parte del instrumental utilizado, lo cual pudo afectar su poder discriminante. Finalmente, se definió un set compuesto de 6 fotografías que lograron una fiabilidad aceptable de 0,709 (Figura 1).
Validez: Con las 6 fotografías se procedió al cálculo de la validez a partir del ACP, para variables categóricas utilizando la rotación Varimax. Para poder aplicar el ACP, se hizo necesario verificar la potencia de las correlaciones, para tal efecto se desarrolló la prueba de Bartlett, tomando como hipótesis nula que las correlaciones no son significativas. Al realizar esta prueba, la hipótesis nula es rechazada (valor $\mathrm{p}=0,009$ ). De manera confirmatoria se determinó el índice KMO para comparar los valores de las correlaciones entre las variables y sus correlaciones parciales, obteniéndose como $\mathrm{KMO}=0,73$; siendo este próximo de 1, por tanto correspondía ser aplicado el ACP.

Luego de realizados estos procedimientos, se observó que existen 3 componentes que juntos explican alrededor del $74 \%$ de la varianza de las respuestas correctas. Al desagregar por componente, se observa que el primero explica un $35 \%$ de la varianza, el segundo aporta con un $22 \%$ y el tercer componente aporta sólo con un 16\% (Tabla 2). Esto se consideró indicativo de que la habilidad de diagnóstico diferencial más bien se mide en dos dimensiones. La primera dimensión estuvo conformada por las Fotografías 1, 5, 7 y 10 , todas lesiones DAI, luego, una segunda conformada por las fotos 8 y 13, ambas lesiones mixtas. De tal forma, es posible establecer que las dos dimensiones corresponden a la habilidad de distinguir DAI, por un lado, y de distinguir UPP, por otro, ya sea que estas últimas se encuentren aisladas o ambas juntas en un mismo paciente.

En la Tabla 3 se presenta la distribución de los profesionales de enfermería según habilidad de diagnóstico diferencial de lesiones DAI, UPP y mixtas. Se observó que alrededor de un $68 \%$ de la muestra estudiada diagnosticó correctamente las fotos 1 y 10, ambas DAI. Esto indica que una mayor cantidad de profesionales logran diagnosticar de forma correcta las DAI comparativamente con las lesiones mixtas. 
Tabla 1. Medidas de resumen, fiabilidad y dificultad de 14 fotografías para la medición de las competencias visuales de profesionales de enfermería para identificar y clasificar lesiones DAI, UPP y mixtas.

\begin{tabular}{ccccccccc}
\hline $\begin{array}{c}\text { Fotografía Tipo de lesión } \\
\text { No } \\
\text { (identificación) }\end{array}$ & $\begin{array}{c}\text { Estadio de avance } \\
\text { (clasificación) }\end{array}$ & Mín & Máx & Media & D.E. & $\begin{array}{c}\text { Alpha de } \\
\text { Cronbach si } \\
\text { se elimina el } \\
\text { elemento }\end{array}$ & $\begin{array}{c}\text { Coeficiente } \\
\text { de } \\
\text { Dificultad } \\
(\text { GCV })\end{array}$ \\
\hline 1 & DAI pura & Severa tipo 2 & 0 & 1 & 0,71 & 0,46 & 0,139 & 0,357 \\
2 & Mixta & UPP no clasif. y DAI tipo 2 & 0 & 1 & 0,36 & 0,488 & 0,011 & 0 \\
3 & UPP pura & Tipo I & 0 & 1 & 0,64 & 0,488 & 0,094 & 0 \\
4 & UPP pura & Tipo II & 0 & 1 & 0,57 & 0,504 & 0,364 & 0 \\
5 & DAI pura & Moderada tipo 1 & 0 & 1 & 0,96 & 0,189 & 0,16 & 0,267 \\
6 & DAI pura & Severa tipo 2 & 0 & 1 & 0,04 & 0,189 & 0,191 & 0,186 \\
7 & DAI pura & Severa tipo 2 & 0 & 1 & 0,61 & 0,497 & 0,019 & 0,795 \\
8 & Mixta & UPP no clasif. y DAI tipo 1 & 0 & 1 & 0,79 & 0,418 & 0,006 & 0,157 \\
9 & UPP pura & Daño tejido profundo & 0 & 1 & 0,71 & 0,46 & 0,34 & 0 \\
10 & DAI pura & Severa tipo 2 & 0 & 1 & 0,75 & 0,441 & 0,119 & 0,527 \\
11 & UPP pura & Tipo III & 0 & 1 & 0,86 & 0,356 & 0,309 & 0,392 \\
12 & Mixta & UPP tipo I y DAI Severa tipo 2 & 0 & 1 & 0,32 & 0,476 & 0,101 & 0,002 \\
13 & Mixta & UPP no clasif. y DAI Severa tipo 2 & 0 & 1 & 0,57 & 0,504 & 0,095 & 0,231 \\
14 & Mixta & UPP no clasif. y DAI Severa tipo 2 & 0 & 1 & 0,82 & 0,39 & 0,124 & 0,082 \\
\hline
\end{tabular}

Alpha de Cronbach $=0,174, \mathrm{n}=28$

Figura 1.6 Fotografías fiables (fiabilidad anidada) utilizadas para medir las habilidades para identificar y clasificar lesiones DAI, UPP y mixtas.

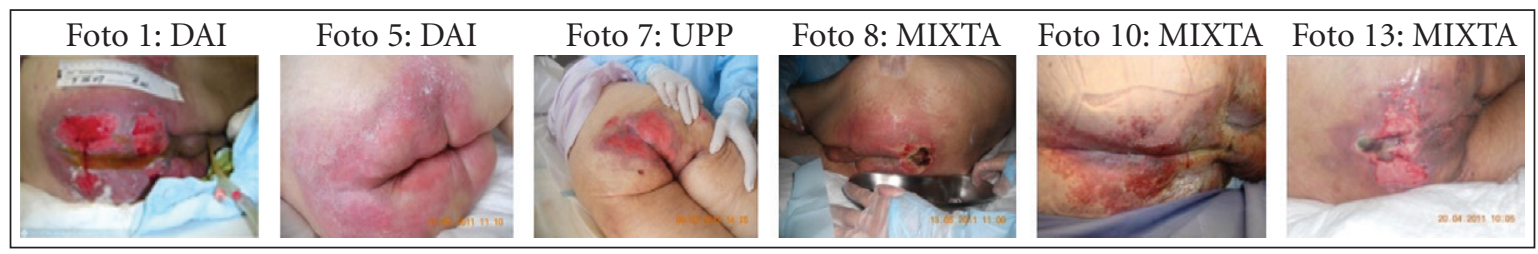

Alpha de Cronbach= 0,709, $\mathrm{n}=28$.

Tabla 2. Matriz de componentes principales extraídos a partir de 6 fotografías fiables para medir las competencias visuales de profesionales de enfermería para identificar y clasificar lesiones DAI, UPP y mixtas, $\mathrm{n}=28$.

\begin{tabular}{llccc}
\hline Fotografía & \multicolumn{3}{c}{ Componente } & $\begin{array}{c}\text { Comunalidades } \\
\text { Extracción }\end{array}$ \\
\hline Foto 1 (DAI) & $\mathbf{0 , 6 0 9}$ & $-0,009$ & 0,466 & 0,589 \\
Foto 5 (DAI) & $\mathbf{0 , 6 0 5}$ & $-0,3$ & 0,467 & 0,674 \\
Foto 7 (DAI) & $\mathbf{0 , 7 4 9}$ & $-0,188$ & $-0,363$ & 0,728 \\
Foto 8 (Mixta) & 0,308 & $\mathbf{0 , 7 7 8}$ & $-0,363$ & 0,832 \\
Foto 10 (DAI) & $\mathbf{0 , 6 8}$ & $-0,377$ & $-0,468$ & 0,823 \\
Foto 13 (Mixta) & 0,505 & $\mathbf{0 , 6 8}$ & 0,267 & 0,789 \\
\hline
\end{tabular}


Tabla 3. Distribución de los profesionales de enfermería según habilidad visual de diagnóstico diferencial de lesiones DAI, UPP y mixtas, $\mathrm{n}=28$.

\begin{tabular}{lrc}
\hline Fotografía & $\mathrm{n}$ & $\%$ \\
\hline Foto 1 (DAI) & 19 & 67,9 \\
Foto 5 (DAI) & 13 & 46,4 \\
Foto 7 (DAI) & 16 & 57,1 \\
Foto 8 (Mixta) & 6 & 21,4 \\
Foto 10 (DAI) & 19 & 67,9 \\
Foto 13 (Mixta) & 3 & 10,7 \\
\hline
\end{tabular}

\section{DISCUSIÓN Y CONCLUSIÓN}

Llama la atención que en la muestra investigada, las fotografías que superan el análisis de fiabilidad son principalmente DAI, mientras que las UPP poseen escaso poder discriminante. Una explicación al respecto se encuentra en la dificultad para distinguir la lesión presentada en cada fotografía. Al respecto, si se observan los valores del coeficiente de dificultad en las fotografías de lesiones UPP eliminadas (fotografías $3,4,9,11$ ), se presentan valores cercanos a 0 , por lo tanto, probablemente para los profesionales de enfermería las UPP son más evidentes y esto afecta el escaso poder discriminante de las fotografías.

Respecto a la dimensionalidad de la habilidad medida, el ACP nos permite establecer que existen dos dimensiones: diagnosticar DAI y diagnosticar UPP. Esto quiere decir que el profesional que logra realizar la distinción entre una y otra lesión, es decir, identificar el tipo de lesión y clasificar su estadio correctamente, ya sea que se presenten por separado o ambas juntas en la misma fotografía, es quien tendría la habilidad de diagnóstico diferencial. Esto es un dato importante para planificar las estrategias de capacitación, las cuales podrían destacar los elementos claves que permiten distinguir un tipo de lesión en comparación con el otro.

En esta investigación se observó que los profesionales logran diagnosticar en mayor proporción las lesiones DAI y en muy baja proporción las lesiones mixtas. Esto es vinculante con estudios previos que indican que las lesiones mixtas son menos diagnosticadas, ya que son difíciles de reconocer (1-3, $12,13,15)$.

Estudios previos indican que las UPP son mejor clasificadas que las DAI $(13,15)$, no obstante, el que en esta investigación alrededor de la mitad de los profesionales fueron capaces de diagnosticar mejor las DAI, tendría su explicación en que este resultado se vincula con las particularidades de la muestra, a saber, profesionales inscritos en un programa de especialización en heridas, de los cuales 22 de ellos tenían al menos una capacitación previa en el área. Dado que la experiencia profesional de las enfermeras es importante en la prevención de UPP (22), podría también la experiencia haber favorecido el mejor rendimiento de la muestra en el diagnóstico de DAI. Este resultado se considera positivo ya que en general las DAI son frecuentemente confundidas. Se plantea entonces que la presencia de capacitaciones previas puede estar aportando mejores habilidades de diagnóstico. Se requiere, en futuros trabajos, contar con una muestra de mayor representatividad estadística y considerar el peso de esta variable.

Cabe señalar el origen de las 6 fotografías que aprueban los procedimientos de fiabili- 
dad y validez estadística. La imagen 1 proviene de la librería de imágenes de la WOCN (23) y la 10 proviene del trabajo de Clark y Black (24), lo que ratifica su utilidad en contextos distintos al de su origen. Las fotografías restantes corresponden a registro propio de Hevia et al. (15).

Los resultados referidos a fiabilidad y validez permiten aportar a la calibración de un instrumento que mide las competencias visuales para realizar un diagnóstico diferencial de lesiones utilizando fotografías. No obstante lo anterior, estos resultados no pretenden ser conclusivos. Una de las limitaciones de este estudio corresponde a las dificultades de acceso y registro fotográfico de los casos, lo cual se traduce en el bajo número de imágenes propuestas y la falta de fotografías que excluyan todo tipo de material (sábanas, pañales, entre otros). Lo anterior vuelve particularmente difícil este campo de investigación, de ahí la naturaleza exploratoria de este trabajo.

Respecto al tamaño de la muestra estudiada, sin duda es pequeño, no obstante entrega importantes antecedentes para la calibración de un instrumento conformado por 6 fotografías que puede ser utilizado para evaluar tanto a enfermeras como a estudiantes de enfermería. Lo anterior, sin duda permite fortalecer la formación de pregrado de la disciplina de enfermería. Se sugiere para futuros trabajos de tipo inferencial, considerar un tamaño de muestra de al menos 51 profesionales (error del 5\%) e incorporar aleatoriedad en el proceso de selección de las unidades muestrales.

Finalmente, una fotografía tampoco es suficiente para evaluar a los profesionales. Se requiere incluir descripciones de las condiciones en las que se encuentra el paciente como inmovilidad, disminución de capacidad sensorial y el tipo de incontinencia.

Los desafíos que enfrenta el país ante el envejecimiento poblacional ponen de relieve la necesidad de contar con profesionales capacitados para distinguir entre DAI y UPP.
Esto permitirá implementar adecuadas medidas de prevención y tratamiento.

\section{REFERENCIAS}

1. Gray M, Beeckman D, Bliss DZ, Fader M, Logan S, Junkin J, et al. Incontinence-Associated Dermatitis: A Comprehensive review and Update. J Wound Ostomy Continence Nurs [Internet]. 2012 [citado 25 sept 2016]; 39(1): 61-74. Disponible en: DOI: 10.1097/WON.0b013e31823fe246

2. Beeckman D, Schoonhoven L, Boucqué H, Van Maele G, Defloor T. Pressure ulcers: e learning to improve classification by nursing students. J Clin Nurs [Internet]. 2008 [citado 25 sept 2016]; 17(13): 1697-1707. Disponible en: DOI: 10.1111/j.1365-2702.2007.02200.x

3. Defloor T, Schoonhoven L, Fletcher J, Furtado K, Heyman H, Lubbers M, et al. Statement of the European-Pressure Ulcer Advisory Panel-Pressure Ulcer Classification. Differentiation Between Pressure Ulcers and Moisture Lesions. J Wound Ostomy Continence Nurs [Internet]. 2005 [citado 25 sept 2016]; 32(5): 302-306. Disponible en: http://recursosbiblioteca.unab.cl:2060/login.aspx?direc$\mathrm{t}=$ true \&db=ccm\&AN=106390439\&lan$\mathrm{g}=$ es\&site=eds-live.

4. Segovia-Gómez T, Bermejo M, García-Alamino J. Úlceras por humedad: conocerlas mejor para poder prevenirlas. Gerokomos. [Internet]. 2012 [citado 25 sept 2016]; 23(3): 137-140. Disponible en: DOI: $10.4321 / \mathrm{s} 1134-$ 928x2012000300009

5. Doughty D, Junkin J, Kurz P, Gray M, Fader M, Bliss DZ, et al. Incontinence-Associated Dermatitis Consensus Statement, Evidence-Based Guidelines for Prevention and Treatment and Current Challenges. J Wound Ostomy Continence Nurs [Internet]. 2012 [citado $25 \mathrm{sept}$ 
2016]; 39(3): 303-315. Disponible en: DOI: 10.1097/WON.0b013e3182549118

6. Voegeli D. Pressure ulcer or moisture lesion-what's the difference? Nursing \& Residential Care [Internet]. 2011 [citado 25 sept 2016]; 13(5): 222-225. Disponible en: doi.org/10.12968/nrec.2011.13.5.222

7. Gray M. Incontinence-Related Skin Damage: Essential Knowledge. Ostomy Wound Management [Internet]. 2007 [citado 25 sept 2016]; 53(12): 28-32. Disponible en: http://www.o-wm.com/content/incontinence-related-skin-damage-essential-knowledge.

8. Gray M, Bliss DZ, Doughy BD, Ermer-Seltum JA, Kennedy- Evans KL, Palmer MH. Incontinence-associated Dermatitis. A consensus. J Wound Ostomy Continence Nurs. 2007; 34(1): 45-54.

9. Black J, Baharestani M, Cuddigan J, Dorner B, Edsberg L, Langemo D, et al. National Pressure Ulcer Advisory Panel's Updated Pressure Ulcer Staging System. Urologic Nursing [Internet]. 2007 [citado 25 sept 2016]; 27(2): 144-156. Disponible en: http://www.nursingcenter.com/pdfjournal?AID=714719\&an=00129334-20 0705000-00012\&Journal_ID=54015\&Issue_ID $=714697$

10. Beeckman D, Van Lancker A, Van Hecke A, Verhaeghe S. A Systematic Review and Meta-Analysis of Incontinence-Associated Dermatitis, Incontinence and Moisture as Risk Factor for Pressure Ulcer Development. Res Nurs Health [Internet]. 2014 [citado 25 sept 2016]; 37(3): 204-218. Disponible en: DOI: 10.1002/ nur.21593.

11. Defloor T, Schoonhoven L, Katrien V, Weststrate J, Myny D. Reliability of the European Pressure Ulcer Advisory Panel classification system. J Adv Nurs [Internet]. 2006 [citado 25 sept 2016]; 54(2): 189-98. Disponible en: DOI: 10.1111/j.1365-2648.2006.03801.x

12. Beeckman D, Schoonhoven L, Fletcher J, Furtado K, Gunningberg L, Heyman C, et al. EPUAP classification system for pressure ulcers: European reliability study. J Adv Nurs [Internet]. 2007 [citado 25 sept 2016]; 60(6): 682-691. Disponible en: DOI: 10.1111/j.1365-2648.2007.04474.x.

13. Beeckman D, Schoonhoven L, Fletcher J, Furtado K, Heiman H, Paquay L, et al. Pressure ulcers and incontinence-associated dermatitis: effectiveness of the Pressure Ulcer Classification education tool on classification by nurses. Qual Saf Health Care [Internet]. 2010 [citado 7 jun 2016]; 19(5): e3. Disponible en: DOI:10.1136/qshc.2008.028415.

14. Baumgarten M, Margolis DJ, Selekof JL, Moye N, Jones P, Shardell M. Validity of pressure ulcer diagnosis using digital photography. Wound Repair Regen [Internet]. 2009 [citado 7 jun 2016]; 17(2): 287-90. Disponible en: DOI: 10.1111/j.1524-475X.2009.00462.x

15. Hevia H, Ríos L, Viveros F. Quantitative study of visual nursing competence in Chile to identified and classified incontinence-associated dermatitis, pressure ulcers and mixed lesions. WCET Journal. 2015; 35(4): 30-35.

16. González R, Mora L, Celis J, Matiz G. Dermatitis asociada a incontinencia en adultos: un problema sin definición, revisión sistemática. Rev Fac Med [Internet]. 2015 [citado 7 jun 2016]; 63(2): 199-208. Disponible en: http://www. scielo.org.co/scielo.php?script=sci_arttext\&pid=S0120-00112015000200004\&l$\mathrm{ng}=\mathrm{en} \& \mathrm{tlng}=\mathrm{en}$.

17. Marco I, González-Romá V, Benito J. Teoría de respuesta al ítem y análisis factorial confirmatorio: dos métodos para analizar la equivalencia psicométrica en la traducción de cuestionarios. Psicothema. 2000; 12: 540-544.

18. Robins J. Optimal structural nested models for optimal sequential decisions. En: Lin D, Heagerty P, editor. Proceedings of the second Seattle Symposium in Biostatistics; 20-21 Nov 2000; Seattle. New 
York: Springer; 2004. p. 189-326.

19. Navarro J, Casas G, González E. Análisis de componentes principales y análisis de regresión para datos categóricos. Aplicación en la presión arterial. Revista de Matemática: Teoría y Aplicaciones [Internet]. 2010 [citado 23 dic 2017]; 17(2): 199-230. Disponible en: DOI: https://doi. org/10.15517/rmta.v17i2.2128

20. Pérez R, Lera L, Boquet A. El doble análisis en componentes principales para datos categóricos y su aplicación en un estudio de migración. Revista Colombiana de Estadística [Internet]. 2006 [citado 23 dic 2017]; 29(1): 17-34. Disponible desde: http://www.scielo.org. co/scielo.php?script=sci_arttext\&pi$\mathrm{d}=\mathrm{S} 0120-17512006000100002 \& 1 n$ $\mathrm{g}=\mathrm{en} \& \mathrm{nrm}=$ iso

21. González J, Carvajal C, Viveros F. Coeficientes edumétricos para la validez y dificultad de un test: Propuesta. Estud. Peda- góg. 2016; 42(3): 467-481.

22. Barker A, Kamar J, Tyndall T, White L, Hutchinson A, Weller C, et al. Implementation of pressure ulcer prevention best practice recommendations in acute care: an observational study. Int Wound J [Internet]. 2013 [citado 27 oct 2016]; 10(3): 313-320. Disponible en: http://dx.doi.org/10.1111/j.1742-481X.2012.00979.x

23. Wound, Ostomy and Continence Nurses Society. The WOCN image library N.D. [Internet]. Wound, Ostomy and Continence Nurses Society; [citado 26 jun 2016]. Disponible en: http://www.wocn. org/page/ImageLibrary.

24. Clark L, Black J. Keeping the Bariatric Patient's Skin Intact. Bariatric Times [Internet]. 2011 [citado 21 jun 2016]; 8(5): 20-23. Disponible en: http://bariatrictimes.com/keeping-the-bariatric-patient\%E2\%80\%99s-skin-intact/. 